Document downloaded from:

http://hdl.handle.net/10251/122261

This paper must be cited as:

Erdogan, E.; Calabuig, JM.; Sánchez Pérez, EA. (2018). Convolution-continuous bilinear operators acting on Hilbert spaces of integrable functions. Annals of Functional Analysis. 9(2):166-179. https://doi.org/10.1215/20088752-2017-003/1

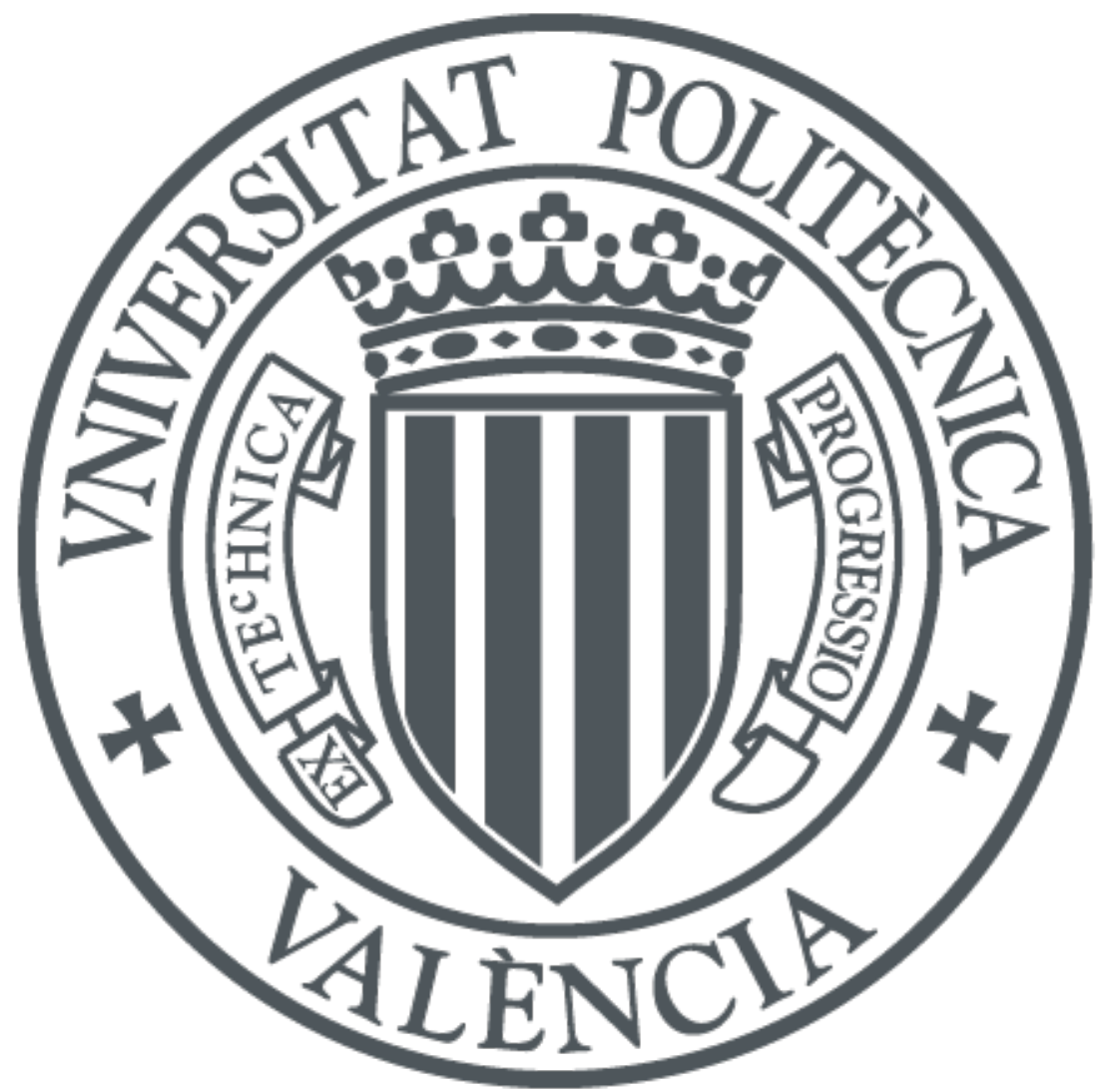

The final publication is available at

http://doi.org/10.1215/20088752-2017-003/1

Copyright Duke University Press

Additional Information 


\title{
CONVOLUTION-CONTINUOUS BILINEAR OPERATORS ACTING IN HILBERT SPACES OF INTEGRABLE FUNCTIONS
}

\author{
EZGI ERDOĞAN,${ }^{1}$ JOSE M. CALABUIG,${ }^{2}$ and ENRIQUE A. SÁNCHEZ PÉREZ, ${ }^{2 *}$
}

\begin{abstract}
We study bilinear operators acting in a product of Hilbert spaces of integrable functions that are 0 -valued for couples of functions whose convolution equals 0 , that we call convolution continuos bilinear maps. We prove a factorization theorem for them, showing that they factor through $\ell^{1}$. We present also some applications for the case when the range space has some relevant properties, as the Orlicz or the Schur properties. We prove that $\ell^{1}$ is the only Banach space for which there is a norming bilinear map which equals 0 exactly in the couples of functions whose convolution is 0 . We also show some examples and applications to generalized convolution.
\end{abstract}

\section{IntroduCtion}

A main outcome of the Classical Harmonic Analysis is the deep result that establishes that the convolution * of two functions in $L^{2}(G)$ of a compact group $G$ has absolutely convergent Fourier series, and functions having this property are exactly the ones that can be written as convolution of two functions in $L^{2}(G)$. Moreover, if we write $\mathfrak{R}(G)$ for the space of functions with absolutely convergent Fourier series, and we define its norm as the $\ell^{1}$-norm of the corresponding Fourier coefficients, the resulting space is isometric to $\ell^{1}$. Its proof goes back to Hewitt and former results, and can be already found in the classical book by Hewitt and Ross ([12]). Also, the characterization of convolution by means of its properties Copyright 2016 by the Tusi Mathematical Research Group.

Date: Received: xxxxxx; Revised: yyyyyy; Accepted: zzzzzz.

${ }^{*}$ Corresponding author.

2010 Mathematics Subject Classification. Primary 47H60; Secondary 43A25, 46 G25.

Key words and phrases. convolution, bilinear operator, factorization, Fourier transform, summability. 
as a bilinear operator acting in spaces of continuous functions is due to Edwards, who published in the classical paper [7] that certain commutation properties for a positive bilinear map imply that it is in fact the convolution operator. On the other hand, some new linearization procedures for analyzing summability and other properties of multilinear maps have been recently introduced. Concretely, factorization of bilinear maps through linear operators acting in classical Banach spaces has proven to be a useful technique for such an analysis (see for instance [13] and the references therein).

Bringing together these ideas, in this paper we study bilinear maps acting in products of $L^{2}$-spaces that equal 0 on convolution-null couples of functions. We will say that these bilinear operators are *-continuous, providing classical and current examples. We show that this apparently weak requirement for a bilinear map implies that it has to be necessarily the composition of convolution with a linear operator. Our main result (Theorem 3.1) proves this fact, opening in this way the door for providing some applications that have the specificity of convolution as essential foundation.

Among them, we prove a characterization of $\ell^{1}-$ up to isomorphisms - as the unique space in which there exists a norming bilinear map with the same nullcouples that convolution (Corollary 4.5). We will give also some results about summability and topological properties of *-continuous maps when some Banach space properties are assumed on the range space $Y$. In particular, we consider finite cotype, the Orlicz property and the Schur property for $Y$, showing that under these assumptions both summability and compactness properties improve.

Finally we explain some consequences of our results in a more applied context. We provide some applications to what are known as generalized convolutions of integral transforms of Fourier type. This topic has been recently developed to provide a unified context for studying integral equations of convolution type (see [9] and the references therein). 


\section{Preliminaries}

Let $X_{1}, X_{2}, Y$ be Banach spaces. We will say that a continuous bilinear map $B: X_{1} \times X_{2} \rightarrow Y$ is weakly compact if the set $B\left(B_{X_{1}} \times B_{X_{2}}\right) \subset Y$ is relatively weakly compact. As usual, we will write $B_{Y}$ for the closed unit ball of the Banach space $Y$. If $A \subseteq Y$, we will denote by $\bar{A}$ the (norm) closure of $A$ in $Y$.

We will say that the bilinear map $B$ is equivalently norming (just "norming" for short) for $Y$ if there is a constant $k>0$ such that

$$
B_{Y} \subseteq k \overline{B\left(B_{X_{1}} \times B_{X_{2}}\right)} .
$$

In the same direction, we will say that a set $A \subseteq\left(B_{X_{1}} \times B_{X_{2}}\right)$ is norming if

$$
B_{Y} \subseteq k \overline{B(A)}
$$

Let $1 \leqslant p, q \leqslant \infty$ and let $X$ and $Y$ be Banach spaces. As usual, we write $L(X, Y)$ for the space of linear and continuous operators from $X$ to $Y$. Recall that an operator $T: X \rightarrow Y$ is said to be $(p, q)$-summing if there is a constant $k>0$ such that for every $x_{1}, \ldots, x_{n} \in X$,

$$
\left(\sum_{i=1}^{n}\left\|T\left(x_{i}\right)\right\|_{Y}^{p}\right)^{1 / p} \leqslant k \sup _{x^{*} \in B_{X^{*}}}\left(\sum_{i=1}^{n}\left|\left\langle x_{i}, x^{*}\right\rangle\right|^{q}\right)^{1 / q} .
$$

We will write $\Pi_{p, q}(X, Y)-\Pi_{p}(X, Y)$ if $p=q$-for the operator ideal of all $(p, q)$-summing operators (see $[5, \S 32])$.

Through all the paper we will consider bilinear maps $B: L^{2}(\mathbb{T}) \times L^{2}(\mathbb{T}) \rightarrow Y$. A relevant example of such a bilinear operator is the convolution map, that is defined for Banach spaces $Y$ containing the space of absolutely convergent Fourier series $\mathfrak{R}(\mathbb{T})$. If $G$ is an locally compact abelian group with Haar measure $\eta$, convolution is defined by the formula

$$
f * g(x)=\int_{G} f(x-y) g(y) d \eta(y), \quad f, g \in L^{1}(G)
$$


and is well-defined and continuous among several domain and range function spaces of integrable functions. The study of the properties of convolution as a bilinear map — and in fact, its characterization by means of these propertiesgoes back to Edwards. In his early paper [7], it is shown that the behavior with respect to invariance with respect to translations and other essential properties for a positive bilinear map actually determines that it must be the convolution operator ([7, Th. 1]; see also $[8, \S 3.1])$. In this paper, the domain space is assumed to be a space of continuous functions with compact support, but the main idea is the same that we develope in the present work; much more is nowadays known about the topic.

We will say that a bilinear map $B: L^{2}(\mathbb{T}) \times L^{2}(\mathbb{T}) \rightarrow Y$ is continuous with respect to convolution ("*-continuous" for short) if for every $f, g \in L^{2}(\mathbb{T})$,

$$
f * g=0 \quad \text { implies } \quad B(f, g)=0 \text {. }
$$

Also, we will say that such a bilinear map is $*$-equivalent if $f * g=0$ if and only if $B(f, g)=0$ for every $f, g \in L^{2}(\mathbb{T})$.

Let us recall some Banach space properties that we will use in the paper; $\ell^{1}$ satisfies all of them.

A Banach space $Y$ has the Dunford-Pettis property if every weakly compact linear operator $T: Y \rightarrow Z$ into another Banach space $Z$ - or only for $Z=c_{0}$ is completely continuous (that is, it transforms weakly compact sets in $Y$ into norm-compact sets in $Z$, see for example $[6, \S \mathrm{VI}])$.

A Banach space $Y$ has the Schur property if weakly convergent sequences and norm convergent sequences coincide in it. The results on this property that we use can be found in [14]; the interested reader can find updated information about variants of this property in [15]. A Banach space has the Orlicz property if the identity map in it is $(2,1)$-summing. 
Let us finish this section by providing some information about the space $\mathfrak{R}(G)$ of functions with absolutely summing Fourier coefficients.

Consider the family $\mathfrak{R}(G)$ of the linear combinations (closure) of continuous positive-definite functions on a topological group $G$. Recall that a function $\phi$ defined on $G$ is said to be positive-definite if the inequality $\sum_{n, m=1}^{N} c_{n} \overline{c_{m}} \phi\left(x_{n}-\right.$ $\left.x_{m}\right) \geqslant 0$ holds for every choice of $x_{1}, x_{2}, . ., x_{N}$ in $G$ and for every choice of complex numbers $c_{1}, c_{2}, \ldots, c_{N}$. It is known that, this family and the space of functions with absolutely summing Fourier coefficiants are exactly same for a compact group $G$ $[12,34.13]$.

For a compact group $G, L^{2}(G) * L^{2}(G)$ gives the space $\mathfrak{R}(G)([12,34.16])$. Moreover, if $G$ is a compact abelian group, $L^{2}(G) * L^{2}(G)$ gives the space of functions which has absolutely convergent Fourier series. Under pointwise operations and with an appropriate norm, $\mathfrak{R}(G)$ is a commutative Banach algebra $([12,34.34(\mathrm{a})])$ with unit, and so for the compact abelian group $G$ with character group $\Gamma$ we get that $\mathfrak{R}(G)$ is isomorphic to the Banach algebra $\ell_{1}(\Gamma)$ via the Fourier transform (see also $[12,34.7]$ ). Recall that the Fourier transform ${ }^{\wedge}$ is a linear isometry from $L^{2}(G)$ onto $L^{2}(\Gamma)$ and the inverse Fourier transform is a linear isometry from $L^{2}(\Gamma)$ onto $L^{2}(G)$; these two transformations are inverses of each other $([12,31.18])$.

In particular, consider the compact abelian group $G$ as $\mathbb{T}$ - the real line mod $2 \pi$ - Then $\mathfrak{R}(\mathbb{T})$ is the unital Banach algebra known as the Wiener algebra. By the isomorphism given by Fourier transform, it is isomorphic to the Banach algebra $\ell^{1}(\mathbb{Z})$.

It must be noted here the obvious fact that the range of a bilinear map is not in general a linear space. However, convolution is also special as bilinear map concerning this aspect. Indeed, as we said before for a compact abelian group $G$ we have that $L^{2}(G) * L^{2}(G)=\mathfrak{R}(G)$ holds, and so $L^{2}(G) * L^{2}(G)$ is a linear space. 
Let us recall also the following known fact about the normability of convolution for the algebra $\mathfrak{R}(G)$. This result is fundamental for our purposes.

Remark 2.1. Let $G$ be a compact abelian group. Convolution is norming for $\mathfrak{R}(G)$ with equivalence constants equal to one. That is, $*\left(B_{L^{2}(G)} \times B_{L^{2}(G)}\right)=B_{\mathfrak{R}(G)}$. Following the notation of [12] recall that the norm in $\mathfrak{R}(G)$ is defined by

$$
\|f\|_{\varphi_{1}}:=\|\hat{f}\|_{1}, \quad f \in \mathfrak{R}(G)
$$

Proof. If $(f, g) \in B_{L^{2}(G)} \times B_{L^{2}(G)}$, we get $\|f * g\|_{\varphi_{1}} \leqslant\|f\|_{2}\|g\|_{2} \leqslant 1$ (see for example $[12,34.14])$. So, $f * g \in B_{\mathfrak{R}(G)}$. For the converse inequality, assume $f \in B_{\mathfrak{R}(G)}$.

Then there are $h, g \in L^{2}(G)$ such that $f=h * g$; this fact can be found in $[12,34.15$ (i)]. By using $[12,34.15$ (iv)], we also obtain that these functions $h$ and $g$ can be chosen in such a way that $1 \geqslant\|f\|_{\varphi_{1}}=\|g\|_{2}^{2}=\|h\|_{2}^{2}$, that is $(h, g) \in B_{L^{2}(G)} \times B_{L^{2}(G)}$.

\section{A COMmutative FACTORIZATION Diagram THROUGH CONVOLUtion}

This section is devoted to show our main result: a factorization theorem for *-continuous bilinear operators. In order to motivate it, we start the analysis by providing some examples of this class of operators. For the aim of clarity, we will write our results for $\mathbb{T}$; the same proofs work for any compact abelian group $G$ with the usual changes.

(1) The first example is constructed by using translation invariant linear operators, - sometimes called convolution operators or multipliers - : they are the operators that commute with translations. The bibliography on this topic is deep and wide, we mention here only the classical paper by Cowling and Fournier [4]. Thus, consider an operator $T: L^{2}(\mathbb{T}) \rightarrow L^{2}(\mathbb{T})$ that satisfies that $T f * g=T(f * g)$. For example, we can take a convolution operator $T_{k}: L^{2}(\mathbb{T}) \rightarrow L^{2}(\mathbb{T})$ with convolution kernel $k \in L^{2}(\mathbb{T})$, that is 
$T_{k} f:=k * f$. Consider now the bilinear map $B_{k}: L^{2}(\mathbb{T}) \times L^{2}(\mathbb{T}) \rightarrow L^{2}(\mathbb{T})$ defined by convolution of $B_{k}(\cdot, \cdot):=T_{k}(\cdot) *(\cdot)$. Then we have

$$
B_{k}(f, g)=T_{k}(f) * g=(k * f) * g=k *(f * g)=T_{k}(f * g),
$$

for $f, g \in L^{2}(\mathbb{T})$. Although for the convolution map defined in products of spaces of continuous functions, these arguments for translation invariant bilinear maps can already be found in the paper by Edwards [7]; see the proof of Proposition 1 in this paper. If $P(D)$ is a linear partial differential operator, bilinear maps as $P(D)(f * g)$ are also usual in applications of the harmonic analysis.

(2) Consider a Banach space $Z$ and a Bochner 2-integrable function $\Phi$ : $[0,2 \pi] \rightarrow Z$. Consider the vector-valued-kernel bilinear operator $B$ : $L^{2}(\mathbb{T}) \times L^{2}(\mathbb{T}) \rightarrow Z$ defined by

$$
B(f, g):=\int_{0}^{2 \pi} \int_{0}^{2 \pi} \Phi(x) f(x-y) g(y) d y d x .
$$

Indeed, it can be written as $(f, g) \mapsto \int_{0}^{2 \pi} \Phi(x)(f * g)(x) d x$, and then it is 0 when $f * g=0$.

Theorem 3.1. Let $\mathbb{T}$ be the real line $\bmod 2 \pi$ and let $Y$ be an arbitrary Banach space. For a bilinear continuous operator $B: L^{2}(\mathbb{T}) \times L^{2}(\mathbb{T}) \rightarrow Y$, the following statements are equivalent:

(i) $B$ is *-continuous.

(ii) There is a linear and continuous map $T: \mathfrak{R}(\mathbb{T}) \rightarrow Y$ such that $B=T \circ *$.

Proof. Assume first $B$ is $*$-continuous. Since the linear map ${ }^{\smile}: \ell^{2}(\mathbb{Z}) \rightarrow L^{2}(\mathbb{T})$ is an isometric isomorphism, we can define the bilinear operator

$$
\widetilde{B}\left(\left(a_{n}\right),\left(b_{n}\right)\right):=B\left(\left(a_{n}\right)^{\smile},\left(b_{n}\right)^{\smile}\right), \quad\left(a_{n}\right),\left(b_{n}\right) \in \ell^{2}(\mathbb{Z}),
$$


which clearly provides $\widetilde{B}(\hat{f}, \hat{g})=B(f, g)$ and the commutativity of the diagram

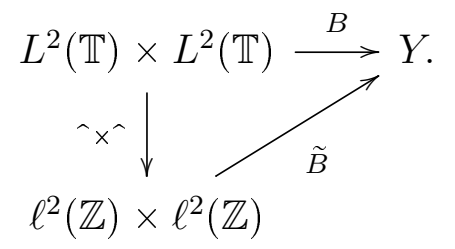

Now, we prove that there is a bounded linear map $\widetilde{T}: \ell^{1}(\mathbb{Z}) \rightarrow Y$ such that the following diagram commutes

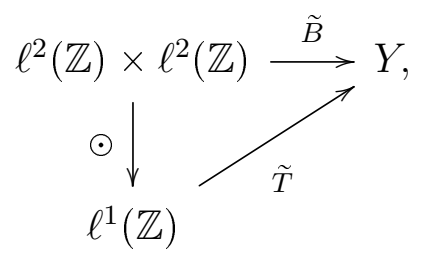

where $\odot$ is the pointwise product of sequences.

For each $N \in \mathbb{N}$ we define the linear map $\widetilde{T}_{N}: \ell^{1}(\mathbb{Z}) \rightarrow Y$ by

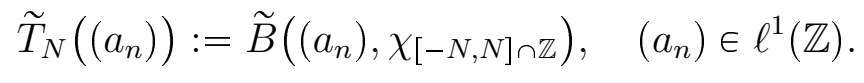

We claim that, since $B$ is $*$-continuous, we have that $\widetilde{B}\left(\chi_{\{i\}}, \chi_{\{j\}}\right)=0$ when $i \neq j$. Indeed, since

$$
0=\chi_{\{i\}} \cdot \chi_{\{j\}}=\widehat{\widehat{\chi_{\{i\}}}} \cdot \widehat{\widehat{\chi_{\{j\}}}}=\left(\overline{\chi_{\{i\}}} * \overline{\chi_{\{j\}}}\right) \hat{,}
$$

the *-continuity of $B$ together with $(3.2)$ gives $\widetilde{B}\left(\chi_{\{i\}}, \chi_{\{j\}}\right)=B\left(\widetilde{\chi_{\{i\}}}, \overline{\chi_{\{j\}}}\right)=0$. Using this remark it is easy to see that

$$
\widetilde{T}_{N}\left(\left(a_{n}\right)\right)=\sum_{|j| \leqslant N} a_{j} \widetilde{B}\left(\chi_{\{j\}}, \chi_{\{j\}}\right)
$$

Therefore

$$
\left\|\widetilde{T}_{N}\left(\left(a_{n}\right)\right)\right\|_{Y} \leqslant \sum_{|j| \leqslant N}\left\|a_{j} \widetilde{B}\left(\chi_{\{j\}}, \chi_{\{j\}}\right)\right\|_{Y}=\sum_{|j| \leqslant N}\left\|\widetilde{B}\left(a_{j} \chi_{\{j\}}, \chi_{\{j\}}\right)\right\|_{Y}
$$




$$
\begin{aligned}
& \leqslant\|\widetilde{B}\| \sum_{|j| \leqslant N}\left\|a_{j} \chi_{\{j\}}\right\|_{\ell^{2}(\mathbb{Z})}\left\|\chi_{\{j\}}\right\|_{\ell^{2}(\mathbb{Z})} \\
& =\|\widetilde{B}\| \sum_{|j| \leqslant N}\left|a_{j}\right| \leqslant\|\widetilde{B}\|\left\|\left(a_{n}\right)\right\|_{\ell^{1}(\mathbb{Z})},
\end{aligned}
$$

and so $\widetilde{T}_{N}$ is continuous; in fact the family $\left\{\widetilde{T}_{N}: N \in \mathbb{N}\right\}$ is uniformly bounded, since $\left\|\widetilde{T}_{N}\right\| \leqslant\|\widetilde{B}\|$ for all $N \in \mathbb{N}$. Moreover, for each fixed $\left(a_{n}\right) \in \ell^{1}(\mathbb{Z}),\left(\widetilde{T}_{N}\left(\left(a_{n}\right)\right)\right)$ is a Cauchy sequence in the Banach space $Y$, and so it is convergent. Indeed, for a given $\varepsilon>0$, there exists $k \in \mathbb{N}$ such that $\sum_{|j|>N}\left|a_{j}\right|<\varepsilon /\|\widetilde{B}\|$ for all $N \geqslant k$. By using (3.5) we have that for all $M>N \geqslant k$,

$$
\begin{aligned}
\left\|\widetilde{T}_{M}\left(\left(a_{n}\right)\right)-\widetilde{T}_{N}\left(\left(a_{n}\right)\right)\right\|_{Y} & =\left\|\sum_{|j| \leqslant M} a_{j} \widetilde{B}\left(\chi_{\{j\}}, \chi_{\{j\}}\right)-\sum_{|j| \leqslant N} a_{j} \widetilde{B}\left(\chi_{\{j\}}, \chi_{\{j\}}\right)\right\|_{Y} \\
& \leqslant \sum_{N<|j| \leqslant M}\left\|\widetilde{B}\left(a_{j} \chi_{\{j\}}, \chi_{\{j\}}\right)\right\|_{Y} \\
& \leqslant\|\widetilde{B}\| \sum_{|j|>N}\left|a_{j}\right|<\varepsilon .
\end{aligned}
$$

Therefore by using Banach-Steinhaus theorem — the Uniform Boundedness Principlethe map $\widetilde{T}: \ell^{1}(\mathbb{Z}) \rightarrow Y$ given by

$$
\widetilde{T}\left(\left(a_{n}\right)\right):=\lim _{N \rightarrow \infty} \widetilde{T}_{N}\left(\left(a_{n}\right)\right)
$$

is linear and bounded. Finally, by the continuity of $\widetilde{B}$ and again (3.5) we have

$$
\begin{aligned}
\widetilde{B}\left(\left(a_{n}\right),\left(b_{n}\right)\right) & =\sum_{j=-\infty}^{\infty} a_{j} b_{j} \widetilde{B}\left(\chi_{\{j\}}, \chi_{\{j\}}\right) \\
& =\lim _{N \rightarrow \infty} \sum_{|j| \leqslant N} a_{j} b_{j} \widetilde{B}\left(\chi_{\{j\}}, \chi_{\{j\}}\right) \\
& =\lim _{N \rightarrow \infty} \widetilde{T}_{N}\left(\left(a_{n} b_{n}\right)\right)=\widetilde{T} \circ \odot\left(\left(a_{n}\right),\left(b_{n}\right)\right),
\end{aligned}
$$

and so the commutativity of (3.3) follows.

On the other hand, given a linear map $\widetilde{T}: \ell^{1}(\mathbb{Z}) \rightarrow Y$ we can use that the Fourier transform ${ }^{\wedge}: \mathfrak{R}(\mathbb{T}) \rightarrow \ell^{1}(\mathbb{Z})$ is an isometric isomorphism (see [12, Cor. 
34.7]) to define an operator $T: \mathfrak{R}(\mathbb{T}) \rightarrow Y$ by

$$
T(f):=\widetilde{T}(\hat{f}), \quad f \in \mathfrak{R}(\mathbb{T})
$$

This gives the factorization

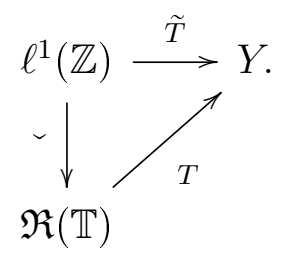

Finally, the classical identity $\widehat{f * g}=\widehat{f} \cdot \widehat{g}$ that works in general for $f, g \in L^{1}(\mathbb{T})$ allows to write $*=^{\smile} \circ \odot \circ\left({ }^{\wedge} x^{\wedge}\right)$. Hence, we obtain the commutativity of the diagram

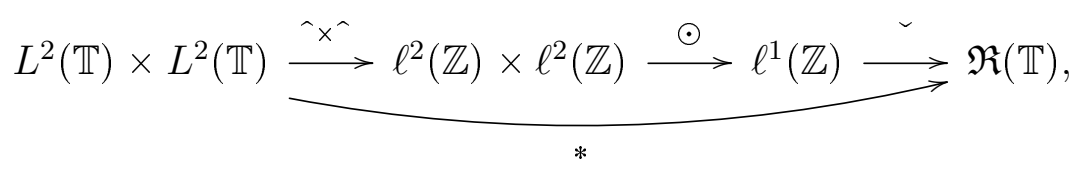

and (ii) holds.

Conversely, assume that there is a linear and continuous operator $T: \mathfrak{R}(\mathbb{T}) \rightarrow$ $Y$ such that $B=T \circ *$. Then if $f * g=0$ obviously $B(f, g)=T(f * g)=0$ and $B$ is *-continuous. The proof is done.

Remark 3.2. Seeing the proof of our result we can give an explicit formula for the operator $T$ in terms of the classical Dirichlet kernel. We claim that the map $T$ of the Theorem is given by:

$$
T(f)=\lim _{N \rightarrow \infty} B\left(f, D_{N}\right)
$$

where $D_{N}$ stands for the Dirichlet kernel which is given by the formula:

$$
D_{N}(x)=\sum_{|j| \leqslant N} e^{i j x}
$$


Indeed just observe that

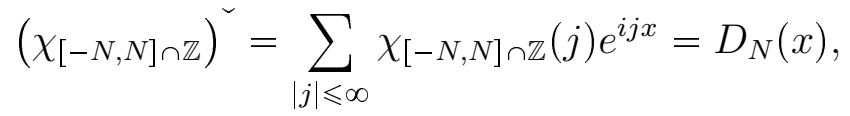

and use (3.7), (3.6), (3.4) and (3.1) to obtain:

$$
\begin{aligned}
T(f) & =\tilde{T}(\hat{f})=\lim _{N \rightarrow \infty} \widetilde{T}_{N}(\hat{f})=\lim _{N \rightarrow \infty} \tilde{B}\left(\hat{f}, \chi_{[-N, N] \cap \mathbb{Z})}\right. \\
& =\lim _{N \rightarrow \infty} B\left(f,\left(\chi_{[-N, N] \cap \mathbb{Z})}\right)=\lim _{N \rightarrow \infty} B\left(f, D_{N}\right) .\right.
\end{aligned}
$$

Actually, putting together the commutativity of diagrams (3.2), (3.3), (3.8) and (3.9) we have proof that:

Corollary 3.3. Let $\mathbb{T}$ be the real line $\bmod 2 \pi$ and let $Y$ be an arbitrary Banach space. For a bilinear continuous operator $B: L^{2}(\mathbb{T}) \times L^{2}(\mathbb{T}) \rightarrow Y$, the following statements are equivalent:

(i) $B$ is *-continuous.

(ii) There are linear and bilinear continuous operators such that the following diagram commutes

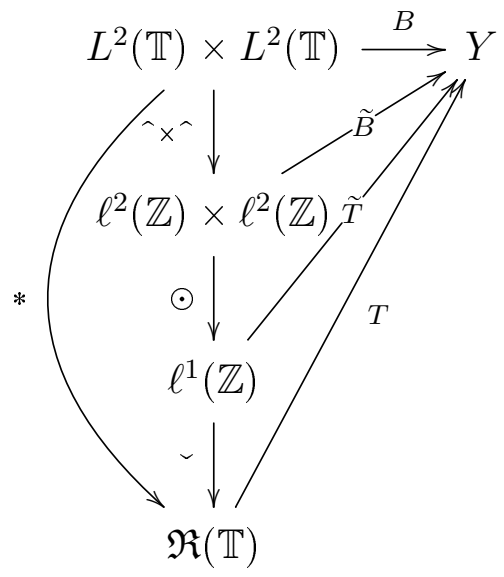

where ${ }^{\wedge}$ and ${ }^{`}$ stand for the Fourier and the Inverse Fourier transforms, respectively, and $\odot$ is the pointwise product of sequences. 
We show now two examples of *-continuous bilinear operators concerning two recent developments in bilinear Fourier analysis.

(A) Let us explain some relations of our class with a genuine bilinear version of convolution, that is given by the so-called translation invariant bilinear operators. A considerable effort has been made recently for understanding this class of maps in the setting of the multilinear harmonic analysis; we refer to [10] and the references therein for information on the topic. They are given — in the case we consider $\mathbb{R}$ as measurable space and the operator is defined by a non-negative regular Borel measure $\mu$ - by the formula

$$
B_{\mu}(f, g):=\int_{\mathbb{R}} \int_{\mathbb{R}} f(x-y) g(x-z) d \mu(y, z), \quad f, g \in L^{2}(\mathbb{R})
$$

(see [10] and the references therein). We consider the "compact group version" of this definition with a slight modification. Take $\mu=k(z) d y d z$ for $k \in L^{2}[0,2 \pi]$ and consider the map

$$
\begin{aligned}
& B_{k}(f, g):=\int_{0}^{2 \pi} \int_{0}^{2 \pi} f(y-x) g(x-z) k(z) d y d z \\
& =\int_{0}^{2 \pi} f(y-x)(k * g)(x) d y, \quad f, g \in L^{2}[0,2 \pi]
\end{aligned}
$$

Using this, and if $\Psi$ is a $Z$-valued Bochner 2-integrable function $-Z$ is a Banach space - we can define the $Z$-valued kernel bilinear map by

$$
B_{\Psi, k}(f, g)=\int_{0}^{2 \pi} \Psi(y)\left(\int_{0}^{2 \pi} f(y-x)(k * g)(x) d x\right) d y, \quad f, g \in L^{2}[0,2 \pi]
$$

Clearly,

$$
B_{\Psi, k}(f, g)=\int_{0}^{2 \pi} \Psi(y)(k *(f * g))(y) d y
$$

and this is 0 if $f * g=0$.

(B) Let $1<p<\infty$ and consider the continuous bilinear map $u: \ell^{p} \times \ell^{p^{\prime}} \rightarrow \ell^{1}$ given by the pointwise product $u\left(\left(a_{i}\right),\left(b_{i}\right)\right):=\left(a_{i}\right) \odot\left(b_{i}\right)=\left(a_{i} b_{i}\right) \in \ell^{1}$. We 
will use for this example the $u$-convolution for spaces of Bochner integrable functions defined by Blasco in [1] (see also [2,3]). Following [1] and the notation in this paper, the $u$-convolution can be defined as a bilinear map $*_{u}: L^{1}\left(\mathbb{T}, \ell^{p}\right) \times L^{1}\left(\mathbb{T}, \ell^{p^{\prime}}\right) \rightarrow L^{1}\left(\mathbb{T}, \ell^{1}\right)$ by the formula

$$
\phi *_{u} \psi(t)=\int_{0}^{2 \pi} u\left(\phi\left(e^{i s}\right), \psi\left(e^{i(t-s)}\right)\right) \frac{d s}{2 \pi} \in L^{1}\left(\mathbb{T}, \ell^{1}\right),
$$

for $\phi \in L^{1}\left(\mathbb{T}, \ell^{p}\right), \psi \in L^{1}\left(\mathbb{T}, \ell^{p^{\prime}}\right)$. Consider now to sequences of integrable functions $\left(k_{i}\right)$ and $\left(v_{i}\right)$ and assume that the linear maps $T_{1}: L^{2}(\mathbb{T}) \rightarrow$ $L^{1}\left(\mathbb{T}, \ell^{p}\right)$ and $T_{2}: L^{2}(\mathbb{T}) \rightarrow L^{1}\left(\mathbb{T}, \ell^{p^{\prime}}\right)$ given by

$$
T_{1}(f)(w):=\sum_{i=1}^{\infty}\left(k_{i} * f\right)(w) e_{i} \in \ell^{p}, \quad T_{2}(g)(w):=\sum_{i=1}^{\infty}\left(v_{i} * g\right)(w) e_{i} \in \ell^{p^{\prime}},
$$

are well-defined for all $f, g \in L^{2}(\mathbb{T})$ and continuous. We consider the bilinear map $B:=*_{u} \circ\left(T_{1}, T_{2}\right): L^{2}(\mathbb{T}) \times L^{2}(\mathbb{T}) \rightarrow L^{1}(\mathbb{T}, Z)$. Let us show that it is *-continuous. Indeed, for a fixed couple of functions $f, g \in L^{2}(\mathbb{T})$, we have

$$
\begin{gathered}
B(f, g)(t)=\int_{0}^{2 \pi}\left(\sum_{i=1}^{\infty}\left(k_{i} * f\right)\left(e^{i s}\right)\left(v_{i} * g\right)\left(e^{i(t-s)}\right) e_{i}\right) \frac{d s}{2 \pi} \\
\left.=\sum_{i=1}^{\infty}\left(\int_{0}^{2 \pi}\left(k_{i} * f\right)\left(e^{i s}\right)\left(v_{i} * g\right)\left(e^{i(t-s)}\right)\right) \frac{d s}{2 \pi}\right) e_{i} \in \ell^{1} .
\end{gathered}
$$

Thus, $B(f, g)=\sum_{i=1}^{\infty}\left(k_{i} * v_{i}\right) *(f * g) e_{i}$, and so it is *-continuous.

\section{Properties of *-CONTINUOUS Bilinear operators AND Applications}

Some direct consequences on the properties of *-continuous bilinear maps can be fixed by using some classical properties. We will analyze separately the main two cases that are reasonable to consider in our context: when $Y$ is a reflexive space, and when $Y$ is a Banach space with the Schur property. In the first one, it will be shown that $*$-continuous operators have good summability properties in case $Y$ has some suitable geometric properties. The second one - that regards 
topological properties - will provide some information in the case that $B$ is weakly compact. We will finish the section - and the paper - by showing an application of our results to what is called generalized convolution.

\subsection{Summability properties of *-continuous bilinear maps: Hilbert spaces,}

finite cotype spaces and $\ell^{p}$-spaces. Consider a $*$-continuous bilinear map $B: L^{2}(\mathbb{T}) \times L^{2}(\mathbb{T}) \rightarrow Y$. In this case, the factorization given by Theorem 3.1 gives that for all $f_{1}, \ldots, f_{n}, g_{1}, \ldots, g_{n} \in L^{2}(\mathbb{T})$,

$$
\sum_{i=1}^{n}\left\|B\left(f_{i}, g_{i}\right)\right\|_{Y} \leqslant k \sum_{i=1}^{n}\left\|\hat{f}_{i} \cdot \hat{g}_{i}\right\|_{\ell^{1}}=k \sum_{i=1}^{n} \sum_{j=1}^{\infty}\left|a_{j}^{i} b_{j}^{i}\right|
$$

where $\left(a_{j}^{i}\right)$ and $\left(b_{j}^{i}\right)$ are the sequences of Fourier coefficients of $f_{i}$ and $g_{i}$, respectively. This summability can be improved when we consider as $Y$ some particular spaces.

The first application that we will show is when $Y$ is a Hilbert space $H$ for instance $H=L^{2}(\mathbb{T})$ - . In this case an integral domination can be even obtained. By Grothendieck's Theorem we have that $L\left(\ell^{1}, H\right)=\Pi_{1}\left(\ell^{1}, H\right)$, and so we directly obtain the following

Corollary 4.1. If $H$ is a Hilbert space and $B: L^{2}(\mathbb{T}) \times L^{2}(\mathbb{T}) \rightarrow H$ is *continuous, then $B$ factors through a summing operator $T$ as $B=T \circ *$. Consequently, there is a constant $k>0$ such that the following equivalent assertions hold.

(i) For $f_{1}, \ldots, f_{n}, g_{1}, \ldots, g_{n} \in L^{2}(\mathbb{T})$,

$$
\sum_{i=1}^{n}\left\|B\left(f_{i}, g_{i}\right)\right\|_{H} \leqslant k \sup _{\varphi \in B_{\ell^{\infty}}} \sum_{i=1}^{n}\left|\left\langle\widehat{f}_{i} \cdot \widehat{g}_{i}, \varphi\right\rangle\right|=k \sup _{\left(\varphi_{j}\right) \in B_{\ell^{\infty}}} \sum_{i=1}^{n}\left|\sum_{j=1}^{\infty} a_{j}^{i} b_{j}^{i} \varphi_{j}\right|,
$$

where $\left(a_{j}^{i}\right)$ and $\left(b_{j}^{i}\right)$ are the sequences of Fourier coefficients of $f_{i}$ and $g_{i}$, respectively. 
(ii) For $f, g \in L^{2}(\mathbb{T})$,

$$
\|B(f, g)\|_{H} \leqslant k \int_{B_{\ell^{\infty}}}|\langle\hat{f} \cdot \hat{g}, \varphi\rangle| d \eta(\varphi)=k \int_{B_{\ell^{\infty}}}\left|\sum_{j=1}^{\infty} a_{j} b_{j} \varphi_{j}\right| d \eta(\varphi),
$$

where $\eta$ is a regular probability measure on the unit ball of $\ell^{\infty}$ given by the Pietsch Domination Theorem, and $\left(a_{j}\right)$ and $\left(b_{j}\right)$ are the sequences of Fourier coefficients of $f$ and $g$, respectively.

Some similar (but weaker) results can also be obtained if we consider some cotype-related properties for the space $Y$. For instance, recall that cotype 2 for a Banach space implies the Orlicz property (see [5, 8.9]); if we ask $Y$ to have the Orlicz property, we get a domination for any *-continuous bilinear map as follows: for $f_{1}, \ldots, f_{n}, g_{1}, \ldots, g_{n} \in L^{2}(\mathbb{T})$,

$$
\left(\sum_{i=1}^{n}\left\|B\left(f_{i}, g_{i}\right)\right\|_{Y}^{2}\right)^{1 / 2} \leqslant k \sup _{\varepsilon_{i} \in\{-1,1\}}\left\|\sum_{i=1}^{n} \varepsilon_{i} \hat{f}_{i} \cdot \hat{g}_{i}\right\|_{\ell^{1}}
$$

The second case that we may consider is the one given when $Y$ is an $\ell^{p}$-space. We have that for $1 \leqslant p \leqslant \infty$ and $r$ such that $1 / r=1-|1 / p-1 / 2|, L\left(\ell^{1}, \ell^{p}\right)=$ $\Pi_{r, 1}\left(\ell^{1}, \ell^{p}\right)$. This result can be found in $[5,34.11]$. Using it we can directly prove the following

Corollary 4.2. Let $1 \leqslant p \leqslant \infty$, and take $r$ such that $1 / r=1-|1 / p-1 / 2|$. Take an *-continuous bilinear map $B: L^{2}(\mathbb{T}) \times L^{2}(\mathbb{T}) \rightarrow \ell^{p}$. Then there is a constant $k>0$ such that for $f_{1}, \ldots, f_{n}, g_{1}, \ldots, g_{n} \in L^{2}(\mathbb{T})$,

$$
\left(\sum_{i=1}^{n}\left\|B\left(f_{i}, g_{i}\right)\right\|_{\ell^{p}}^{r}\right)^{1 / r} \leqslant k \sup _{\varepsilon_{i} \in\{-1,1\}}\left\|\sum_{i=1}^{n} \varepsilon_{i} \hat{f}_{i} \cdot \hat{g}_{i}\right\|_{\ell^{1}}=k \sup _{\left(\varphi_{j}\right) \in B_{\ell^{\infty}}} \sum_{i=1}^{n}\left|\sum_{j=1}^{\infty} a_{j}^{i} b_{j}^{i} \varphi_{j}\right|
$$

where $\left(a_{j}^{i}\right)$ and $\left(b_{j}^{i}\right)$ are the sequences of Fourier coefficients of $f_{i}$ and $g_{i}$, respectively.

Note that for the case $p=2$ we obtain the result given in Corollary 4.1. Other interesting case is the one provided by the classical Littlewood inequality, that 
can be written as $L\left(\ell^{1}, \ell^{4 / 3}\right)=\Pi_{4 / 3,1}\left(\ell^{1}, \ell^{4 / 3}\right)$ (see $\left.[5,34.12]\right)$ : if $B$ is defined on $\ell^{4 / 3}$, we obtain that

$$
\left(\sum_{i=1}^{n}\left\|B\left(f_{i}, g_{i}\right)\right\|_{\ell^{4 / 3}}^{4 / 3}\right)^{3 / 4} \leqslant k \sup _{\varphi \in B_{\ell} \infty} \sum_{i=1}^{n}\left|\left\langle\hat{f}_{i} \cdot \hat{g}_{i}, \varphi\right\rangle\right| .
$$

\subsection{Weak compactness and bilinear maps on spaces with the Schur}

property. In this section we analyze some properties for $Y$ that are typical for non-reflexive spaces, that would imply some concrete consequences on the factorization through the convolution map.

The assumption in this section is that $B: L^{2}(\mathbb{T}) \times L^{2}(\mathbb{T}) \rightarrow Y$ is weakly compact, that is, $B\left(B_{L^{2}(\mathbb{T})} \times B_{L^{2}(\mathbb{T})}\right)$ is a relatively weakly compact set in $Y$.

Let us introduce some notation. We will say that a subset $A \subseteq L^{2}(\mathbb{T}) \times L^{2}(\mathbb{T})$ is *-relatively weakly compact if the set $\{f * g:(f, g) \in A\} \subseteq \Re(\mathbb{T})$ is relatively weakly compact.

The following result shows that *-continuous operators satisfy a certain kind of Dunford-Pettis property.

Corollary 4.3. Let $B: L^{2}(\mathbb{T}) \times L^{2}(\mathbb{T}) \rightarrow Y$ be an $*$-continuous weakly compact bilinear map, and let $A$ be an *-relatively weakly compact set. Then $B(A)$ is relatively compact.

Moreover, if there is a*-relatively weakly compact bilinear operator $B$ for $Y$, then $Y$ is finite dimensional.

Proof. This is just a consequence of the fact that $\ell^{1}$ has the Dunford-Pettis property. Since we have the factorization though $\ell^{1}$ of $B$, and such factorization satisfies that

$$
*\left(B_{L^{2}(\mathbb{T})} \times B_{L^{2}(\mathbb{T})}\right)=B_{\Re(\mathbb{T})}
$$

we have that $\left.\Upsilon^{(*}\left(B_{L^{2}(\mathbb{T})} \times B_{L^{2}(\mathbb{T})}\right)\right)=B_{\ell^{1}}$. Therefore, $T 0^{\smile}: \ell^{1} \rightarrow Y$ is weakly compact. Take an $*$-relatively weakly compact set $A$. We have that $\left.{ }^{\wedge} * A\right)$ is then a relatively weakly compact of $\ell^{1}$. The Dunford-Pettis property of $\ell^{1}$ gives then 
that $T \circ{ }^{\circ} \widehat{\circ} \circ *(A)=T \circ *(A)=B(A)$ is relatively compact. The last statement is then clear.

This theorem can be improved for the case that $Y$ has the Schur property; recall that this means that weakly and norm convergent sequences coincide in it. Notice that there are spaces other than $\ell^{1}$ that have the Schur property (for example, some discrete Nakano spaces, see (IV) in [11]). The same kind of arguments that we use in the following result with a bit more restrictive requirements on $B$ will be used in the next one to prove that then *-continuous bilinear maps can indeed characterize $\ell^{1}$.

Corollary 4.4. Let $B: L^{2}(\mathbb{T}) \times L^{2}(\mathbb{T}) \rightarrow Y$ be an $*$-continuous weakly compact bilinear map. Let $Y$ be a Banach lattice with the Schur property. Then $B\left(B_{L^{2}(\mathbb{T})} \times\right.$ $\left.B_{L^{2}(\mathbb{T})}\right)$ is a relatively compact set in $Y$.

Consequently, if $B$ is norming for $Y$, then $Y$ is finite dimensional.

Proof. We use Theorem 2 in [14], that establishes that a Banach space $Y$ has the Schur property if and only if every weakly compact operator from $\ell^{1}$ to $Y$ is compact. As we shown in the proof of Corollary 4.3 we have that ${ }^{\wedge}\left(*\left(B_{L^{2}(\mathbb{T})} \times\right.\right.$ $\left.\left.B_{L^{2}(\mathbb{T})}\right)\right)=B_{\ell^{1}}$. Therefore, $T \circ^{\smile}: \ell^{1} \rightarrow Y$ is weakly compact, and so the Schur property for $Y$ gives that it is also compact. Then obviously normability of $B$ implies that $Y$ has finite dimension.

Recall that a bilinear map $B$ is *-equivalent if for every $f, g \in L^{2}(\mathbb{T})$,

$$
f * g=0 \quad \Longleftrightarrow \quad B(f, g)=0
$$

Corollary 4.5. A Banach space $Y$ is isomorphic to $\ell^{1}$ if and only if it admits a *-equivalent norming bilinear map $B: L^{2}(\mathbb{T}) \times L^{2}(\mathbb{T}) \rightarrow Y$.

Proof. The direct implication is obvious: if $R: \ell^{1} \rightarrow Y$ is an isomorphism, just take $B=R \stackrel{\wedge}{\circ} \circ$. 
For the converse implication, suppose that $B$ satisfies the requirements. Now take into account that we have a factorization of $B$ as $B=T \circ *$ as a consequence of Theorem 3.1. Since we also have that $B(f, g)=0$ implies $f * g=0$, we have that $T$ (and so $\widetilde{T}$ ) is injective. But $B$ is norming, and so we have that

$$
\widetilde{T}\left(B_{\ell^{1}}\right) \subseteq k B_{Y} \subseteq k K B\left(B_{L^{2}(\mathbb{T})} \times B_{L^{2}(\mathbb{T})}\right)=k K \tilde{T}\left(B_{\ell^{1}}\right)
$$

This gives the result.

\subsection{Applications: *-continuous generalized convolutions. Let us finish} the paper by showing a remark on a new construction that has shown to be useful for applications. It concerns to what is called generalized convolution; the reader can find information about in [9, Def.2.3] (see also the references in this paper for the original definitions). Let $U_{1}, U_{2}$ and $U_{3}$ be linear spaces (may be different) on the same field of scalars and let $V$ be a commutative algebra. Suppose that $K_{1} \in L\left(U_{1}, V\right), K_{2} \in L\left(U_{2}, V\right)$ and $K_{3} \in L\left(U_{3}, V\right)$ are the linear operators from $U_{1}, U_{2}$ and $U_{3}$ to $V$ respectively.

Definition 4.6. (Definition 2.3 in [9]) A bilinear map * : $U_{1} \times U_{2} \rightarrow U_{3}$ is called the convolution with weight-element $\delta$ - an element of the algebra $V$ - for $K_{3}, K_{1}, K_{2}$ (in that order) if the following identity holds:

$$
K_{3}(*(f, g))=\delta K_{1}(f) K_{2}(g)
$$

for any $f \in U_{1}$ and $g \in U_{2}$. The equality above is called the factorization identity of the convolution.

Fix $U_{1}=U_{2}=L^{2}(\mathbb{T}), U_{3}=V=\mathfrak{R}(\mathbb{T})$ and $K_{3}=i d$ and consider $*$ as the usual convolution bilinear map. Let us write now a characterization of when a bilinear map defined as a product in the algebra of two linear operators define a 
generalized convolution associated to *. Indeed, as a consequence of Theorem 3.1 we directly obtain the following

Corollary 4.7. Consider two operators $S_{1}, S_{2}: L^{2}(\mathbb{T}) \rightarrow \mathfrak{R}(\mathbb{T})$ and $\delta \in \mathfrak{R}(\mathbb{T})$, and consider the bilinear map $B: L^{2}(\mathbb{T}) \times L^{2}(\mathbb{T}) \rightarrow \mathfrak{R}(\mathbb{T})$ given by $B(\cdot, \cdot)=$ $\delta S_{1}(\cdot) S_{2}(\cdot)$. Then the following assertions are equivalent.

(i) $B$ is *-continuous.

(ii) There is an operator $T: \mathfrak{R}(\mathbb{T}) \rightarrow \mathfrak{R}(\mathbb{T})$ such that $*$ is a convolution with weight $\delta$ for $T, S_{1}, S_{2}$.

In this case, the factorization identity is $T \circ *=B=\delta S_{1} S_{2}$.

Acknowledgments. The first author was supported by TÜBITAK- The Scientific and Technological Research Council of Turkey. The second author was supported by the Ministerio de Economía, Industria y Competitividad (Spain) under project MTM2014-53009-P. The third author was supported by the Ministerio de Economía, Industria y Competitividad (Spain) under project MTM201677054-C2-1-P.

\section{REFERENCES}

[1] O. Blasco, Convolution by means of bilinear maps, Contemporary Math. 232 (1999) 85-104.

[2] O. Blasco, Bilinear maps and convolutions, Research and Expositions Math. 24 (2000) 45-55.

[3] O. Blasco and J. M. Calabuig, Fourier analysis with respect to bilinear maps, Acta Math. Sin. (Engl. Ser.) 25,4 (2009), 519-530.

[4] M. G. Cowling and John J.F. Fournier, Inclusions and noninclusion of spaces of convolution operators, Trans. Amer. Math. Soc. 221,1 (1976) 59-95.

[5] A. Defant and K. Floret, Tensor norm and operator ideals, North-Holland, Amsterdam, 1993.

[6] J. Diestel and J. J. Uhl, Jr., Vector measures, American Mathematical Society, Providence, R.I., 1977. 
[7] R. E. Edwards, Convolutions as bilinear and linear operators, Canad. J. Math. 16 (1964) 275-285.

[8] R. E. Edwards, Fourier series: a modern introduction. Vol. 1, Grad. Texts in Math., Springer, 1979.

[9] B. T. Giang and M. N. Tuan, Generalized convolutions and the integral equations of the convolution type, Complex Variables and Elliptic Equations 55,4 (2010) 331-345.

[10] L. Grafakos and J. Soria, Translation-invariant bilinear operators with positive kernels, Int. Eq. Op. Th. 66.2 (2010) 253-264.

[11] I. Halperin and H. Nakano, Generalized $l_{p}$ spaces and the Schur property, J. Math. Soc. Japan, 5(1), (1953) 50-58.

[12] E. Hewitt and K. A. Ross, Abstract Harmonic Analysis, Vol. II. Springer, Berlin, 1970.

[13] D. Pellegrino, P. Rueda and E. A. Sánchez-Pérez, Surveying the spirit of absolute summability on multilinear operators and homogeneous polynomials, RACSAM 110,1 (2016) 285-302.

[14] W. Wnuk, Some characterizations of Banach lattices with the Schur property. Rev. Mat. Complut. 2(3) (1989) 217-224.

[15] W. Wnuk, On the dual positive Schur property in Banach lattices. Positivity 17,3 (2013) 759-773.

\footnotetext{
${ }^{1}$ Department of Mathematics, Faculty of Art and Science, University of MarMARA, TR-34722, KADIKÖy, Istanbul, TURKEY

E-mail address: ezgi.erdogan@marmara.edu.tr

${ }^{2}$ Instituto Universitario de Matemática Pura y Aplicada, Universitat Politècnica de València, Camino de Vera s/n. 46022 Valencia. Spain

E-mail address: jmcalabu@mat.upv.es

E-mail address: easancpe@mat.upv.es
} 